\title{
Numerical modelling of landslide-generated waves with the particle finite element method (PFEM) and a non-Newtonian flow model
}

\author{
F. Salazar ${ }^{* *}$, J. Irazábal ${ }^{*}$, A. Larese ${ }^{* \dagger}$, E. Oñate ${ }^{* \dagger}$
}

\begin{abstract}
SUMMARY
Landslide-generated impulse waves may have catastrophic consequences. The physical phenomenon is difficult to model due to the uncertainties in the kinematics of the mobilised material, and to the intrinsic complexity of the fluid-soil interaction. The Particle Finite Element Method (PFEM) [1] is a numerical scheme which has successfully been applied to fluid-structure interaction problems. It uses a Lagrangian description to model the motion of nodes (particles) in both the fluid and the solid domains (the later including soil/rock and structures). A mesh connecting the particles (nodes) is re-generated at every time step, where the governing equations are solved. Various constitutive laws are used for the sliding mass, including rigid solid and Newtonian and non-Newtonian fluids. Several examples of application are presented, corresponding both to experimental tests and to actual full-scale case studies. The results show that the PFEM can be a useful tool for analysing the risks associated to landslide phenomena, providing a good estimate to the potential hazards even for full-scale events. Copyright (c) 2010 John Wiley \& Sons, Ltd.

Received ...
\end{abstract}

KEY WORDS: Landslide generated waves; Particle finite element method; Non-Newtonian fluid; Lagrangian formulation

\footnotetext{
*Centre Internacional de Métodes Numérics en Enginyeria (CIMNE), Barcelona, Spain

†Universidad Politécnica de Cataluña (UPC), Barcelona, Spain

Copyright (c) 2010 John Wiley \& Sons, Ltd.

Prepared using nagauth.cls [Version: 2010/05/13 v2.00]
} 


\section{INTRODUCTION}

Landslides generated in the vicinity of water bodies such as reservoirs, lakes or bays can cause impulse waves with important consequences. Although the probability of occurrence is generally low, many casualties have been produced by this kind of events throughout history [2], [3].

Landslides falling in reservoirs are particularly interesting because water level variations (especially rapid draw-downs) can favour its occurrence [4]. The presence of the reservoir for a long enough time saturates the slopes, what increases the pore pressure and, as a consequence, decreases the effective stress. This destabilising effect is partially compensated by the raise of the total stress due to the hydrostatic pressure generated by the reservoir. A rapid draw-down eliminates the stabilisation in a lapse which is frequently not enough for the pore pressure to be dissipated (this depends on the permeability of the material, as well as on the velocity of the pool level drop, but is quite frequent). In this scenario the risk of landslides increases.

Numerical modelling of this phenomenon is complex because four consecutive events have to be considered: the landslide itself, the impact against the still water, the wave formation and its propagation. Furthermore, there is a high degree of uncertainty in the initial conditions, what implies that some assumptions have to be made on the triggering mechanism, the reservoir level at the time of the event, the degree of fracturing of the mass at impact and the friction coefficient in the slide surface, among others. Finally, actual case studies comprise large domains, what results in high computational cost and resources needed.

In spite of this complexity, an important research effort is being made worldwide in this field. Only as regards the sliding phenomenon, a wide variety of approaches have been applied, in correspondence to the numerous types of failure mechanisms [5]. Landslide-generated waves have been modelled with several numerical techniques. Three-dimensional $(3 D)$ examples are commonly treated with the shallow-water equations (e.g. [6], [7], [8]), although fully $3 D$ approaches have

\footnotetext{
*Correspondence to: Centre Internacional de Métodes Numérics en Enginyeria (CIMNE) C/ Gran Capitán s/n, Edificio C-1. Campus Norte UPC, Barcelona 08034, Spain Tel.: (34) 934016035 
sometimes been used, such as the smooth particle hydrodynamics (SPH) method [3]. Ward and Day [9], [10] presented the simplified "Tsunami balls"method, based on particles representing wave energy, and applied it to simulate landslide case studies at full-scale.

The phenomenon of landslide-generated waves is a paradigmatic example of fluid-soil-structure interaction problems, for whose analysis advanced numerical methods have been developed by the authors' groups [11], [12], [13]. More precisely, the authors have implemented a particular class of Lagrangian formulation for solving problems involving complex interactions between free surface fluids and solids. The so-called the Particle Finite Element Method (PFEM) treats the mesh nodes in the fluid and solid domains as particles which can freely move and even separate from the main fluid domain representing, for instance, the effect of water drops. A mesh connects the nodes discretising the domain where the governing equations are solved using a stabilised version of the Finite Element Method (FEM). Details of the PFEM can be found in [1], [11], [14], [15], [16], [17], [18].

Solids can be modelled in different ways with the PFEM, what makes it quite versatile. Apart from the fixed or moving solid boundaries, interaction between fluids and moving (rigid or elastic) solids can also be computed, as well as the erosion of river beds [19] or even the failure of rock-fill dams due to overtopping [20], [21].

The PFEM has previously been applied to model landslides [22], as well as landslide-generated waves [23], [24]. The results encouraged the development of more appropriate constitutive models to better reproduce the kinematics of landslides of different type. In the current work, a pressure sensitive non-Newtonian Bingham-like model is presented and implemented in a PFEM algorithm. A similar kind of constitutive model was previously used by Pastor and co-workers [25] in the context of depth integrated models but not in Lagrangian finite elements techniques. A stabilised equal order mixed velocity pressure scheme is proposed to solve the non-Newtonian Navier-Stokes equations. An Algebraig Sub-Grid Scale (ASGS) stabilisation technique is employed to overcome the issues related with pressure instabilities. Finally, a second order Bossak time integration scheme is implemented to allow an easy coupling of the model with other structural dynamic solvers. 
The rest of the paper is organised as follows. The non-Newtonian constitutive laws utilised are discussed and the balance equation are presented. Then the weak form of the problem is obtained and the implementation procedure is outlined. The basic ideas of the PFEM are introduced. Finally, several examples of application are described, showing the capabilities of the method for modelling landslide-generated waves.

\section{CONSTITUTIVE MODELS}

One of the intrinsic difficulties in the numerical simulation of landslides is the uncertainty on the kinematics of the unstabilised flowing mass. The geotechnical information on the site may narrow the possibilities, though some aspects remain inevitable unknown. It should be recalled that in the general case, the interest lies in the simulation of hypothetical (i.e. not occurred) events. Previous works based on experimental tests relied on two basic alternatives: rigid solids or granular noncohesive materials. While the former can be numerically modelled as solid boundaries, several options can be chosen for the latter. Whatever the case, the use of an appropriate constitutive law is highly influential in the results [2].

One option worth to be mentioned is the so-called discrete (or distinct) element method (DEM), that is reaching widespread popularity in the computational mechanics community. The basic idea is that every particle is a discrete element interacting with the others considering its mechanical and material properties [13], [26]. While this approach could be adapted to the case of avalanches, it has the disadvantage that the particle size is unknown a priori.

In this work, a continuum approach has been adopted, what requires the choice of an appropriate constitutive law relating the stresses and the strain rate in the landslide mass. A simple option is to model the landslide as a Newtonian fluid with a given density and viscosity [23], [24].

It is commonly accepted that geomaterials can undertake certain degree of stress without large deformations. When the yield stress is reached, the material starts to "flow" until arriving at a stable configuration. There exists a wide category of fluids which exhibits a rigid behaviour until reaching a yield threshold; they are part of the so-called non-Newtonian fluids family [27], [28]. 
The simplest class of non-Newtonian fluids follow a Bingham law, so that they behave as rigid solids for shear stresses below a given threshold (yield stress) and as Newtoninan fluids above it. The one-dimensional $(1 D)$ constitutive relation for a Bingham plastic can be defined as

$$
\begin{array}{ll}
\dot{\gamma}=0 & \text { if } \tau<\tau_{0} \\
\dot{\gamma}=\frac{1}{\mu}\left(\tau-\tau_{0}\right) & \text { if } \tau \geq \tau_{0}
\end{array}
$$

being $\tau_{0}$ the yield stress, $\dot{\gamma}$ the rate of strain, $\mu$ the dynamic viscosity of the equivalent fluid and $\tau$ the dynamic shear stress.

The Bingham bilinear behaviour might induce numerical difficulties, hence smooth laws are usually preferred. In this work, a regularised model as proposed by Papanastasiou [29] and later implemented by Larese et al. [12] is used. Eqs. 1 are regularised as

$$
\tau=\left[\mu+\frac{\tau_{0}}{\dot{\gamma}}\left(1-e^{-m \dot{\gamma}}\right)\right] \dot{\gamma}
$$

where $m$ is a regularisation parameter that controls the approximation to the bilinear model as shown in Figure 1. The apparent viscosity is defined as

$$
\tilde{\mu}(\dot{\gamma})=\mu+\frac{\tau_{0}}{\dot{\gamma}}\left(1-e^{-m \dot{\gamma}}\right)
$$

Eq. 3 solves the problems connected to the singular point of the bilinear model. 


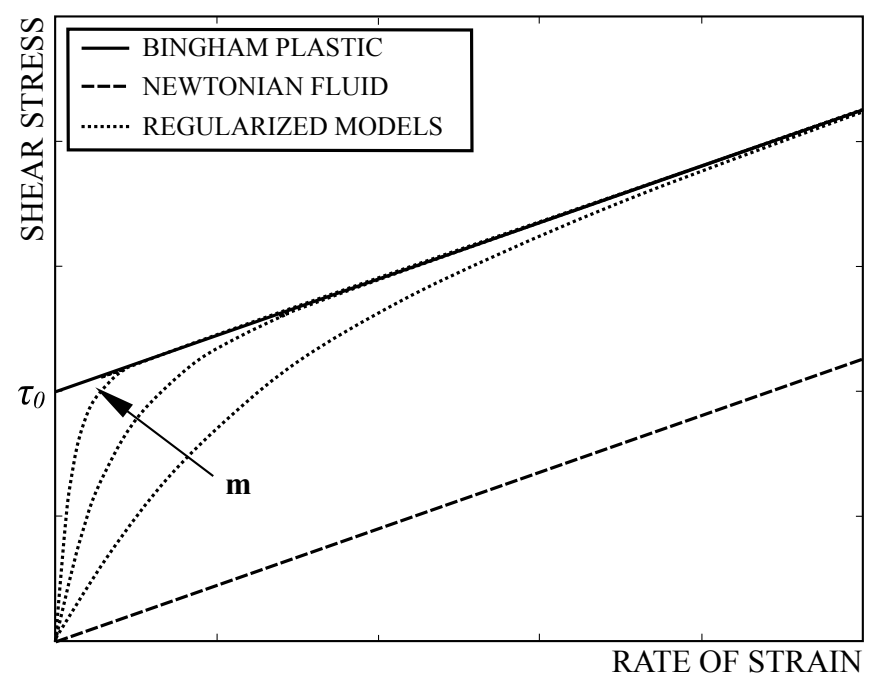

Figure 1. Newtonian and Bingham fluid compared to the regularised model for increasing values of the $m$ parameter. Image taken from [21].

In $3 D$ problems, the stress tensor is represented by

$$
\boldsymbol{\sigma}=-p^{\prime} \mathbf{I}+\boldsymbol{\tau}=-p^{\prime} \mathbf{I}+2 \tilde{\mu} \varepsilon(\mathbf{u}),
$$

where $p^{\prime}$ is the effective pressure that multiplied by the identity matrix I, represents the volumetric part of the model and $\tau$ is the deviatoric part of the stress tensor which is linearly related to the rate of strain $\varepsilon(\mathbf{u})$, where $\mathbf{u}$ is the velocity vector, through the apparent viscosity $\tilde{\mu}$. Therefore in $3 D$, the "equivalent strain rate" $\dot{\gamma}$ and the "yield stress" $\tau_{0}$ are defined as the second invariants of the strain tensor $(\varepsilon)$ and of the deviatoric part of the stress tensor $(\boldsymbol{\tau})$, respectively.

$$
\begin{aligned}
\dot{\gamma} & =\left(\frac{1}{2} \varepsilon: \varepsilon\right)^{\frac{1}{2}} \\
\tau & =\left(\frac{1}{2} \boldsymbol{\tau}: \boldsymbol{\tau}\right)^{\frac{1}{2}}
\end{aligned}
$$

Similarly, Eq. 2 becomes

$$
\boldsymbol{\tau}=2\left[\mu+\frac{\tau_{0}}{\dot{\gamma}}\left(1-e^{-m \dot{\gamma}}\right)\right] \varepsilon(\mathbf{u})
$$

where 


$$
\tilde{\mu}(\dot{\gamma})=\mu+\frac{\tau_{0}}{\dot{\gamma}}\left(1-e^{-m \dot{\gamma}}\right)
$$

This is the approach followed also by Cremonesi et al. [24], that has been implemented in this work and applied in the examples described in Section 6.4. It requires the definition of both the yield stress and the regularisation parameter for each specific case, which can only be done by means of a calibration process.

In an effort to overcome this drawback, Larese et al. [21] developed a model based on the classical Bingham constitutive relation, but using a pressure-sensitive variable yield stress $\tau_{0}$ defined using a Mohr-Coulomb-like failure criteria without cohesion as

$$
\tau_{0}=p^{\prime} \operatorname{tg}(\phi)
$$

where $p^{\prime}$ is the effective pressure and $\phi$ is the internal friction angle. Substituting Eq. 9 into Eq. 7 this becomes

$$
\boldsymbol{\tau}=2\left[\mu+\frac{p^{\prime} \operatorname{tg}(\phi)}{\dot{\gamma}}\left(1-e^{-m \dot{\gamma}}\right)\right] \boldsymbol{\varepsilon}(\mathbf{u})
$$

and the resultant apparent viscosity is, therefore,

$$
\tilde{\mu}(\dot{\gamma})=\mu+\frac{p^{\prime} \operatorname{tg}(\phi)}{\dot{\gamma}}\left(1-e^{-m \dot{\gamma}}\right)
$$

This approach only requires the definition of the internal friction angle of the material, which can be deduced from the geotechnical information.

\section{BALANCE EQUATIONS}

The balance equations are represented by the Navier-Stokes equations for a non-Newtonian fluid.

The present model has been developed in a Lagrangian framework. Hence the mesh velocity vector $\mathbf{u}_{M}$ coincides with the fluid velocity vector $\mathbf{u}$ and, therefore, the convective velocity $\mathbf{a}=\mathbf{u}-\mathbf{u}_{M}$ vanishes. 
Calling $\Omega \subset \mathbb{R}^{d}$ (where $d$ is the space dimension) the structural domain in a time interval $(0, T)$, the Navier-Stokes equations for a non-Newtonian material are

$$
\begin{aligned}
\rho \partial_{t} \mathbf{u}+\nabla p^{\prime}-2 \nabla \cdot \tilde{\mu} \nabla \mathbf{u}-\rho \mathbf{b} & =\mathbf{0} \text { in } \Omega, t \in(0, T), \\
\nabla \cdot \mathbf{u} & =0 \text { in } \Omega, t \in(0, T),
\end{aligned}
$$

where $p^{\prime}$ is the effective pressure and $\rho$ the solid dry density of the material.

The problem is fully defined with the following boundary and initial conditions:

$$
\begin{aligned}
\mathbf{u}(\mathbf{x}, t) & =\mathbf{g}(\mathbf{x}, t) \quad \text { on } \quad \partial \Omega_{D}, \quad t \in(0, T), \\
\mathbf{n} \cdot \boldsymbol{\sigma}(\mathbf{x}, t) & =\mathbf{t}(\mathbf{x}, t) \quad \text { on } \partial \Omega_{N}, \quad t \in(0, T), \\
\mathbf{u}(\mathbf{x}, 0) & =\mathbf{u}_{0}(\mathbf{x}) \quad \text { in } \Omega,
\end{aligned}
$$

\section{WEAK FORM}

The weak form of Eqs. 12 is obtained using a traditional Galerkin formulation [30] and integrating by parts the pressure and viscous terms.

For a fixed $t \in(0, T), \mathbf{u}$ is assumed to belong to the velocity space $\mathcal{V} \in\left[\mathbf{H}^{1}(\Omega)\right]^{d}$ of vector functions whose components and their first derivatives are square-integrable, and $p^{\prime}$ belongs to the pressure space $\mathcal{Q} \in \mathbf{L}_{2}$ of square-integrable functions. w and $q$ are the velocity and pressure weighting functions belonging to velocity and pressure space respectively.

Let $\mathcal{V}_{h}$ be a finite element space to approximate $\mathcal{V}$, and $\mathcal{Q}_{h}$ a finite element approximation to $\mathcal{Q}$. The problem is now finding $\mathbf{u}_{h} \in \mathcal{V}_{h}$ and $p_{h}^{\prime} \in \mathcal{Q}_{h}$ such that

$$
\begin{aligned}
\int_{\Omega} \mathbf{w}_{h} \rho \partial_{t} \mathbf{u}_{h} d \Omega-\int_{\Omega} p_{h}^{\prime} \nabla \cdot \mathbf{w}_{h} d \Omega+\int_{\Omega} \mathbf{w}_{h} \rho \mathbf{u}_{h} \cdot \nabla \mathbf{u}_{h} d \Omega & \\
+2 \int_{\Omega} \nabla \mathbf{w}_{h}: \tilde{\mu} \nabla^{s} \mathbf{u}_{h} d \Omega-\int_{\Omega} \mathbf{w}_{h} \rho \mathbf{b} d \Omega-\int_{\partial \Omega_{N}} \mathbf{w}_{h} \cdot \mathbf{t}_{h} d \Gamma=\mathbf{0} & \forall \mathbf{w} \in \mathcal{V} ; \\
\int_{\Omega} q_{h} \nabla \cdot \mathbf{u}_{h}=0 & \forall q_{h} \in \mathcal{Q}_{h} ;
\end{aligned}
$$




\subsection{Stabilised formulation}

In this work low-order simplicial elements are used with the same linear interpolation for the velocity and pressure values. Hence, as usual for this type of finite element approximation, a stabilisation technique is needed for the pressure. An Algebraic Sub-Grid Space (ASGS) stabilisation technique is employed for that purpose [31]. The stabilised form of the balance equations becomes

$$
\begin{aligned}
\int_{\Omega} \mathbf{w}_{h} \rho \partial_{t} \mathbf{u}_{h} d \Omega-\int_{\Omega} p_{h}^{\prime} \nabla \cdot \mathbf{w}_{h} d \Omega+2 \int_{\Omega} \nabla^{s} \mathbf{w}_{h}: \tilde{\mu} \nabla \mathbf{u}_{h} d \Omega & \\
-\int_{\Omega} \mathbf{w}_{h} \rho \mathbf{b} d \Omega-\int_{\partial \Omega_{N}} \mathbf{w}_{h} \mathbf{t}_{h} d \Gamma+\sum_{e l} \int_{\Omega^{e l}} \tau_{1} \mathcal{P}^{m} \cdot \mathcal{R}^{m} d \Omega=\mathbf{0} \quad & \forall \mathbf{w}_{h} \in \mathcal{V}_{h} ; \\
\int_{\Omega} q_{h} \nabla \cdot \mathbf{u}_{h} d \Omega+\sum_{e l} \int_{\Omega^{e l}} \tau_{2} \mathcal{P}^{c} \cdot \mathcal{R}^{c} d \Omega & =0 \quad \forall q_{h} \in \mathcal{Q}_{h} ;
\end{aligned}
$$

where $\mathcal{P}^{m}, \mathcal{R}^{m}, \mathcal{P}^{c}$ and $\mathcal{R}^{c}$ are defined in Table I.

\subsection{Discretisation procedure}

The matrix form of the stabilised system of equations 15 can be written as:

$$
\left[\begin{array}{cc}
\mathbf{M} & \mathbf{0} \\
\mathbf{0} & \mathbf{0}
\end{array}\right] \cdot\left[\begin{array}{c}
\dot{\mathbf{u}} \\
\dot{\mathbf{p}}
\end{array}\right]+\left[\begin{array}{cc}
\mathbf{K}+\mathbf{S}^{c} & \mathbf{G} \\
\mathbf{D}+\mathbf{S}_{q u} & \mathbf{S}_{q p}
\end{array}\right] \cdot\left[\begin{array}{c}
\mathbf{u} \\
\mathbf{p}
\end{array}\right]=\left[\begin{array}{c}
\mathbf{F} \\
\mathbf{S}_{q}^{f}
\end{array}\right]
$$

where the operators are explicitly written in Table II and the stabilisation operators can be found in Table III.

Eqs. 16 in compact form is

$$
\overline{\mathbf{M}} \dot{\mathbf{v}}+\overline{\mathbf{K}} \mathbf{v}=\mathbf{F}_{e x t} .
$$

where $\mathbf{v}=\left[\begin{array}{c}\mathbf{u} \\ \mathbf{p}\end{array}\right]$ and $\dot{\mathbf{v}}=\left[\begin{array}{c}\dot{\mathbf{u}} \\ \dot{\mathbf{p}}\end{array}\right]$ are the vectors of unknowns. 
Table I. Element stabilising terms in Eq. (15)

Momentum equation

\begin{tabular}{cc}
\hline $\mathcal{P}^{m}$ & $\nabla q_{h}$ \\
$\tau_{1}$ & $\left(\frac{\alpha}{\Delta t}+\frac{4 \tilde{\mu}}{h^{2} \rho}\right)^{-1}$ \\
$\mathcal{R}^{m}$ & $\partial_{t} \mathbf{u}_{h}-\nabla \cdot \frac{\tilde{\mu}}{\rho} \nabla^{s} \mathbf{u}_{h}+\nabla p_{h}^{\prime}-\mathbf{b}$ \\
\hline
\end{tabular}

Continuity equation

\begin{tabular}{lc}
\hline $\mathcal{P}^{c}$ & $\nabla \cdot \mathbf{w}_{h}$ \\
& $\frac{\tilde{\mu}}{\rho}$ \\
$\tau_{2}$ & $\nabla \cdot \mathbf{u}_{h}$ \\
$\mathcal{R}^{c}$ & \\
\hline
\end{tabular}

Table II. Matrices and vectors of the system in Eq. 16 without stabilisation terms.

\begin{tabular}{cc}
\hline Matrix term & Continuum term \\
\hline $\mathbf{M} \dot{\mathbf{u}}$ & $\sum_{e l} \int_{\Omega_{e l}} \mathbf{w}_{h} \rho \partial_{t} \mathbf{u}_{h} d \Omega$ \\
$\mathbf{K u}$ & $2 \sum_{e l} \int_{\Omega_{e l}} \mathbf{w}_{h} \nabla \mathbf{w}_{h}: \tilde{\mu} \nabla \mathbf{u}_{h} d \Omega$ \\
$\mathbf{G} \mathbf{p}$ & $-\sum_{e l} \int_{\Omega_{e l}} p_{h}^{\prime} \nabla \cdot \mathbf{w}_{h} d \Omega$ \\
$\mathbf{D u}$ & $\sum_{e l} \int_{\Omega_{e l}} q_{h} \nabla \cdot \mathbf{u}_{h} d \Omega$ \\
$\mathbf{F}$ & $\sum_{e l} \int_{\Omega_{e l}} \mathbf{w}_{h} \rho \mathbf{b} d \Omega$ \\
$\mathbf{h}$ & $\mathbf{0}$ \\
\hline
\end{tabular}


Table III. Stabilisation matrices and vectors in Eq. 16.

\begin{tabular}{cc}
\hline & Continuity equation \\
\hline Matrix term & Continuum term \\
\hline $\mathbf{S}_{q u} \mathbf{u}$ & $-\sum_{e l} \int_{\Omega_{e l}} \tau_{1} \nabla q_{h} \nabla \cdot \frac{\tilde{\mu}}{\rho} \nabla^{s} \mathbf{u}_{h} d \Omega$ \\
$\mathbf{S}_{q p} \mathbf{p}$ & $\sum_{e l} \int_{\Omega_{e l}} \tau_{s 1} \nabla q_{h} \nabla p_{h}^{\prime} d \Omega$ \\
$\mathbf{S}_{q}^{f}$ & $-\sum_{e l} \int_{\Omega_{e l}} \tau_{s 1} \nabla q_{h} \mathbf{b}_{h} d \Omega$ \\
\hline & Momentum equation \\
\hline $\mathbf{S}^{c} \mathbf{u}$ & $\sum_{e l} \int_{\Omega_{e l}} \tau_{s 2} \nabla \cdot \mathbf{w}_{h} \nabla \cdot \mathbf{u}_{h} d \Omega$ \\
\hline
\end{tabular}

\subsection{Bossak time integration scheme}

A residual based solution strategy is adopted to get the solution at time step $n+1$ and a Bossak time integration scheme is implemented [32], [33]. This common choice in structural problems, is quite unusual in a fluid solver but has been adopted here for future coupling with a structural solver using the same scheme.

The residual (r) of the momentum equations is linearised in time as

$$
\begin{aligned}
\mathbf{r}\left(\mathbf{v}^{n+1-\alpha_{B}}\right)=-\overline{\mathbf{M}} & \left(\frac{1-\alpha_{B}}{\delta \Delta t} \mathbf{v}^{n+1}\right)-\overline{\mathbf{K}} \mathbf{v}^{n+1} \\
& +\mathbf{F}_{e x t}^{n+1}-\overline{\mathbf{M}}\left[\frac{1-\alpha_{B}}{\delta \Delta t} \mathbf{v}^{n}+\frac{\left(1-\alpha_{B}\right)^{2}}{\delta} \dot{\mathbf{v}}^{n}-\alpha_{B} \dot{\mathbf{v}}^{n}\right]
\end{aligned}
$$

where

$$
\alpha_{B} \in\left[-\frac{1}{3}, 0\right], \quad \delta=\frac{1-2 \alpha_{B}}{2}
$$

A residual based predictor multi-corrector strategy can therefore be used to solve for the nodal velocities and the nodal pressures at the end of the time step. Calling $k$ the iteration within the time step the final solution system is

$$
-\underbrace{\frac{\partial \mathbf{r}\left(\mathbf{v}^{n+1, k}\right)}{\partial \mathbf{v}^{n+1}}}_{L H S} \Delta \mathbf{v}^{k}=\underbrace{\mathbf{r}\left(\mathbf{v}^{n+1, k}\right)}_{R H S} ;
$$


The viscous term is the non-linear part of the balance equation. When calculating the LHS of Eq. 20, this is linearised as follows

$$
\left[\mu+\frac{p^{\prime n+1, k} \operatorname{tg}(\phi)}{\dot{\gamma}^{n+1, k}}\left(1-e^{m \dot{\gamma}^{n+1, k}}\right)\right] \nabla^{s} \mathbf{u}^{n+1, k+1} .
$$

Equation 20 is finally solved according to the following steps

1. Predict the nodal unknowns $\mathbf{v}^{\mathbf{n}+1, \mathbf{k}+\mathbf{1}}=\mathbf{v}^{\mathbf{n}+\mathbf{1}, \mathrm{k}}$;

2. Solve the system of Eq. (20) in its residual based form;

3. Update the nodal unknowns $\mathbf{v}^{\mathbf{n}+\mathbf{1}, \mathbf{k}+\mathbf{1}}=\mathbf{v}^{\mathbf{n}+\mathbf{1}, \mathbf{k}}+\Delta \mathbf{v}^{k}$;

\section{Check convergence;}

5. Go back to step 2 till convergence is achieved.

\section{THE PARTICLE FINITE ELEMENT METHOD (PFEM)}

As the domain is expected to undergo severe deformations as the failure progresses, the kinematic model has to adapt dynamically to such deformations. This justifies the preferable choice of a Lagrangian approach. Among the many possible Lagrangian methods, the Particle Finite Element Method (PFEM) has been chosen and implemented for its flexibility and reliability [14], [15]. The PFEM is a numerical method that uses a finite element mesh to discretise the physical domain and to integrate the differential governing equations [17], [15]. In the PFEM the domain is modelled using an Updated Lagrangian Formulation. All the variables are assumed to be known at the current configuration at time $t^{n}$ and they are brought in the next (or updated) configuration at time $t^{n+1}$. The finite element method (FEM) is used to solve the continuum equations in a mesh built up from the underlying nodes (the particles). This is useful to model the separation of solid particles from the bed surface and to follow their subsequent motion as individual particles with a known density, an initial acceleration and a velocity subject to gravity forces.

It is important to underline that in PFEM each particle is treated as a material point characterised by the density of the solid domain to which it belongs to. The global mass is obtained by integrating 
density at the different material points over the domain. The quality of the numerical solution depends on the discretisation chosen as in the standard FEM. Adaptive mesh refinement techniques can be used to improve the solution in zones where large gradients of the fluid or the structural variables occur.

The PFEM is particularly suited for modelling and simulation of landslides and their effect in the surrounding structures, as suggested by the results of previous works [11], [23], [24].

The basic ingredients of the PFEM can be summarised in:

- An Updated Lagrangian kinematical description of the motion;

- A fast remeshing algorithm;

- A boundary recognition method based on the alpha-shape technique [34];

- FEM for the solution of the governing equations;

\subsection{PFEM algorithm}

Considering that the solution at time step $n$ is known, the basic steps of the PFEM algorithm are summarised in the box that follows.

\section{PFEM algorithm}

1. Imposition of the mesh velocity at the calculated time step $n\left(t^{n}\right) \mathbf{u}_{\mathbf{M}}=\mathbf{u}^{\mathbf{n}}$;

2. Movement of the nodes according to the velocity field at $t^{n}$;

3. Laplacian smoothing $\ddagger$;

4. Remeshing step;

5. $\alpha$-shape to detect the boundaries of the domain;

6. Solve the monolithic system;

7. Back to step 1.

\footnotetext{
¥The Laplacian smoothing is a geometrical technique that allows a more homogeneous redistribution of the nodes inside the analysis domain without changing the connectivities between nodes 


\section{APPLICATIONS}

\subsection{Rigid solid in two dimensions}

In the first example, the experiments performed by Sælevik et al. [35] were modelled. The experimental facility comprised a set of solid boxes which were pushed towards a still mass of water in a prismatic channel ( $25 \mathrm{~m}$ long, $0.51 \mathrm{~m}$ wide and $1.0 \mathrm{~m}$ deep). The initial water depth was $0.6 \mathrm{~m}$, and the evolution of the free surface was recorded at three gauges, as depicted in Figure 2.

Three scenarios were run in the laboratory, with different geometry and velocity of the sliding blocks. Scenarios 2 and 3 were reproduced with the PFEM (Table IV).

Preliminary results of the numerical simulation of this experiment, correspondent to scenario 1 , were presented in [23]. Although the wave period was accurately simulated, a deviation of $15 \%$ versus the experimental value was registered for the maximum wave height.

In that case, the impact velocity in the numerical model was set to match that measured in the experiment, and the blocks were assumed to move under the gravity force afterwards. Moreover, the blocks were considered to stop once the front reached the channel bottom, thus neglecting the effect of their movement along the horizontal surface.

In this work, the other two scenarios run by Sælevik et al. have been simulated, with a modification in the kinematics of the blocks. More precisely, they were allowed to go along the channel bottom, until all became horizontal. The velocity until the front reached the channel bottom was assumed to be constant and equal to that reported in [35] at impact. Then, the blocks moved with constant deceleration until they stop.

The experiment was modelled in $2 D$, with an initial mesh of 3-noded linear triangles. The size of the elements was limited to $1 \mathrm{~cm}$. 


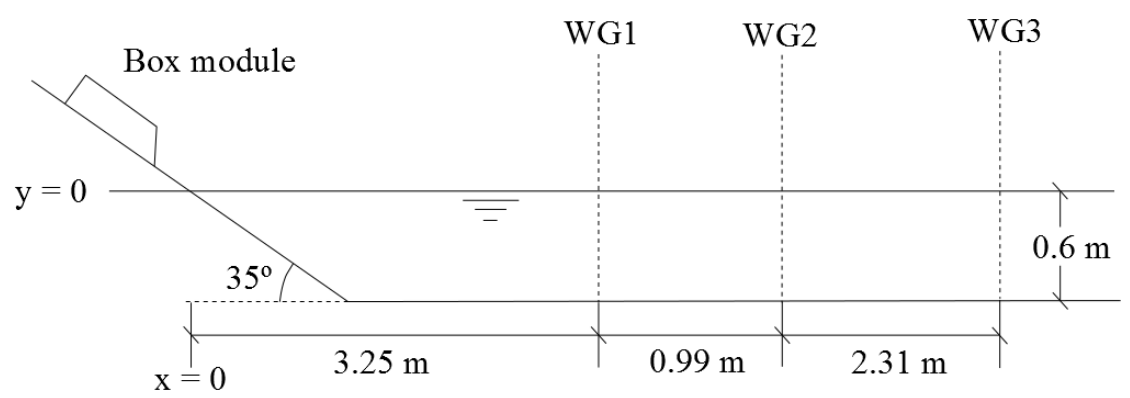

Figure 2. Scheme of the experiments conducted by Sælevik et al. [35].

Table IV. Summary of the slide parameters in the cases considered, which correspond to the scenarios 2 and 3 defined by Sælevik et al. [35].

\begin{tabular}{cccc}
\hline Scenario & Box height & Box length & Impact velocity $(\mathrm{m} / \mathrm{s})$ \\
\hline 2 & $0.16 \mathrm{~m}$ & $2 \times 0.5 \mathrm{~m}$ & 3.38 \\
3 & $0.12 \mathrm{~m}$ & $2 \times 0.5 \mathrm{~m}$ and $1 \times 0.6 \mathrm{~m}$ & 3.56 \\
\hline
\end{tabular}

Figure 3 shows the comparison between the numerical and the experimental results. It can be seen that the difference for both scenarios and the three measurement locations range from 0 to $1 \mathrm{~cm}$, i.e., not larger than the mesh size. As regards to the arriving time, the maximum wave height was registered slightly in advance in the numerical model. 


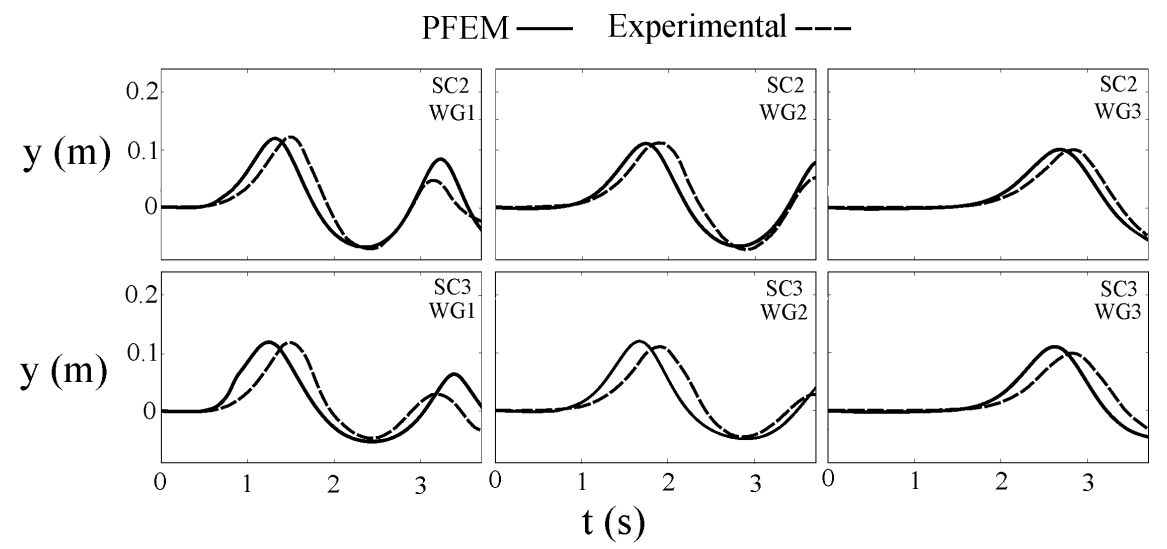

Figure 3. Comparison between the results of the physical model (taken from Sælevik et al. [35]) and the PFEM calculation. Top: scenario 2. Bottom: scenario 3 (see Table IV). The location of the wave gauges is depicted in figure 2

\subsection{Two-dimensional simulation of the Lituya bay landslide}

The Lituya Bay landslide took place in Alaska on July 9, 1958, when an earthquake triggered a large landslide that mobilised around 90 million tons of rock. The impact against the still water generated a wave that reached a maximum height of $524 \mathrm{~m}$ on the opposite shore. It was widely analysed, due to its relevance and to the fact of being well documented, as compared to other events. Some authors attempted to simulate it with numerical models (e.g. Quecedo et al. [36]), while others applied experimental techniques, such as Fritz et al. [37], whose results were chosen to compare those obtained with the PFEM in this work.

The experiment [37] aimed to reproduce the Lituya Bay landslide in a prismatic channel at 1:675 scale, assuming that the lateral wave spreading could be neglected without loss of accuracy. 


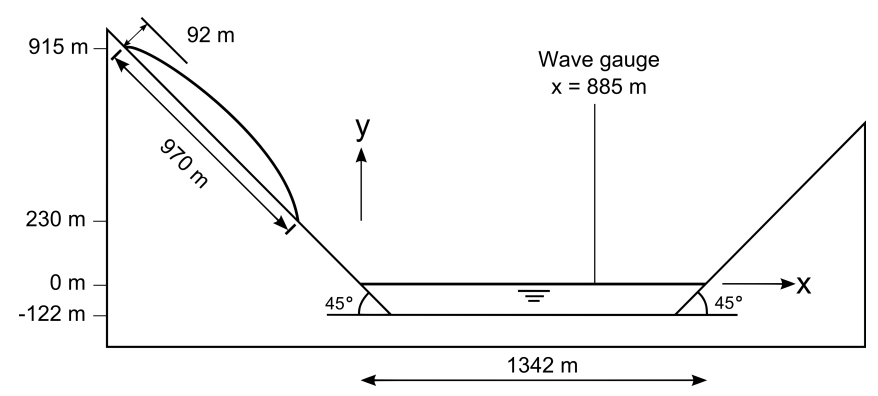

Figure 4. Lituya Bay landslide in two-dimensions (adapted from Fritz et al. [37]).

In the experiment the slide was modelled using an artificial granular material with an average diameter of $4 \mathrm{~mm}$ and a grain density of $2,640 \mathrm{~kg} / \mathrm{m}^{3}$, according to the estimated density of the actual material. The bulk density was $1,620 \mathrm{~kg} / \mathrm{m}^{3}$, with a porosity of $39 \%$. The effective internal friction angle was $43^{\circ}$.

The numerical model of Quecedo et al. [36] took into account the three fluids involved (air, water and landslide) separately, which were considered immiscible. The calculation was repeated for several values of the density and the friction coefficient, concluding that the results did not vary substantially.

The authors performed a similar work previously with the PFEM, in which the sliding mass was considered as a viscous fluid [23]. The wave formation and propagation was qualitatively reproduced, although relevant differences were found in terms of the landslide kinematics, maximum wave height and run-up.

In this work, the slide was modelled as a Bingham fluid with a variable yield stress, as described in Section 2. Hence, it was characterised on the basis of the bulk density $\left(1,620 \mathrm{~kg} / \mathrm{m}^{3}\right)$ and the internal friction angle $\left(43^{\circ}\right)$ of the material used in the experiments. The kinematic viscosity was assumed to be that of water $\left(10^{-6} \mathrm{~m}^{2} / \mathrm{s}\right)$, once confirmed that it had minor effect in the results (see section 6.4). The regularisation parameter $m$ was also of lower importance in this case, provided that the landslide moved with high velocity and thus it behaved under high strain rate (Figure 1). A sufficiently high value was chosen $(3,000)$. The water was modelled as a Newtonian fluid (density: $1,000 \mathrm{~kg} / \mathrm{m}^{3}$; kinematic viscosity: $10^{-6} \mathrm{~m}^{2} / \mathrm{s}$ ), and no-slip condition was imposed at the boundaries. 
The slide in the experiment was accelerated with an ad-hoc device, so that the velocity at impact reached $110 \mathrm{~m} / \mathrm{s}$. The same condition was applied in the numerical model.

Figures 5 and 6 show some snapshots of equivalent time steps of the experimental and the numerical models. Some aspects should be noted:

- The different stages of the phenomenon, as described by Fritz et al. [37], could be neatly observed in the numerical results: “(a) Slide impact and penetration (Figure 5 a), flow separation (Figure $5 \mathrm{~b}$ ), cavity formation (Figure $5 \mathrm{c}, \mathrm{d}, \mathrm{e}, \mathrm{f}$ ) while slide penetration velocity exceeds wave propagation velocity, and (b) cavity collapse (Figure $6 \mathrm{a}, \mathrm{b}$ ), slide run-out along channel bottom, slide detrainment and deposition (Figure $6 \mathrm{c}, \mathrm{d}, \mathrm{e}, \mathrm{f}$ ) as the wave overtakes the landslide and propagates out of the impact area". The air was not considered in this work, as can be observed in Figure 6 i, j, k, l. The inclusion of the air is however possible in the PFEM and this will be the objective of future work in this field.

- The maximum wave elevation at the gauge location $(\mathrm{x}=885 \mathrm{~m})$ was $138 \mathrm{~m}$ in the numerical model, $9 \%$ lower than the observed experimentally $(152 \mathrm{~m})$.

- The boundary of the slide in the numerical model shows differences versus the experimental snapshots. The front separates from the channel bottom, the total length is higher during the slide, and lower at the end of the event. However, the position of the front follows closely the result observed in the laboratory up to $t=16.50 \mathrm{~s}$. Furthermore, the variable-yield Bingham model used reproduced the measured slide motion neatly better than previous works ([23], [36]). 

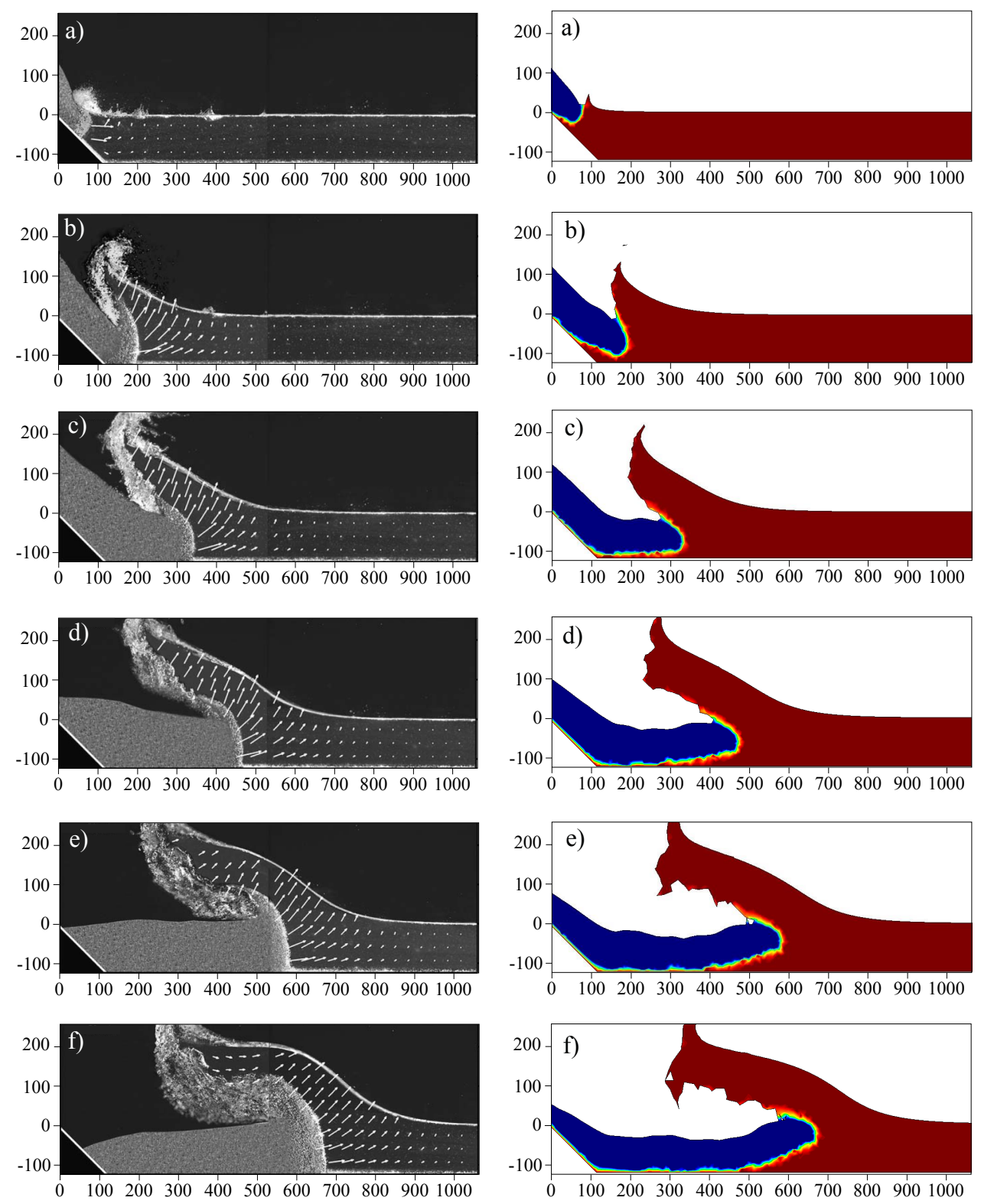

(a) Results taken from [37]

(b) PFEM

Figure 5. Lituya Bay landslide. Comparative snapshots of the experiment by Fritz et al. [37] (left) and the results using PFEM and a variable-yield Bingham model (right). The first image was taken $0.75 \mathrm{~s}$ after impact. The time increment between images is $1.75 \mathrm{~s}$. 

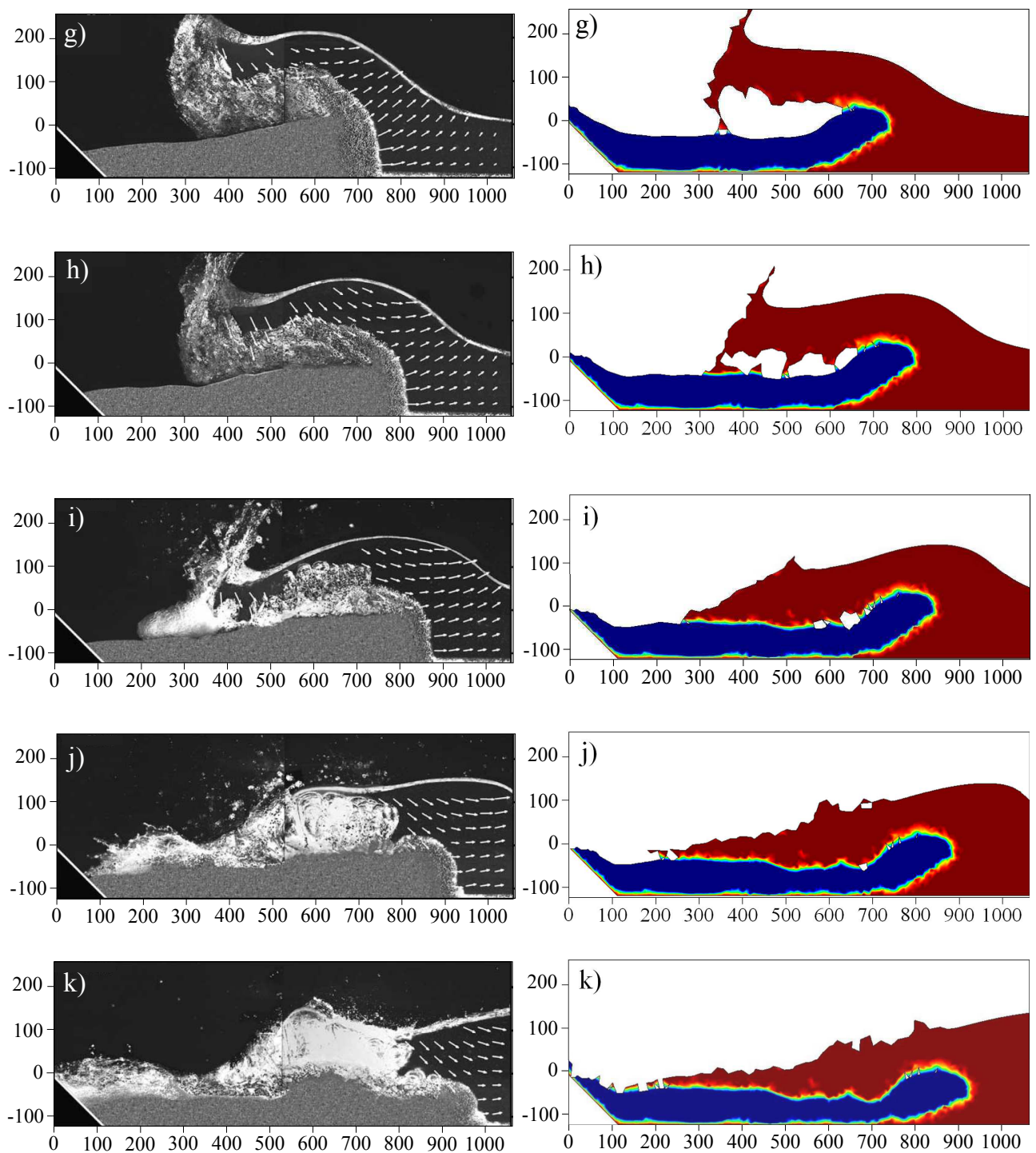

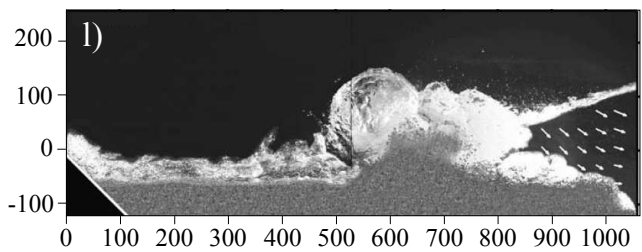

(a) Results taken from [37]

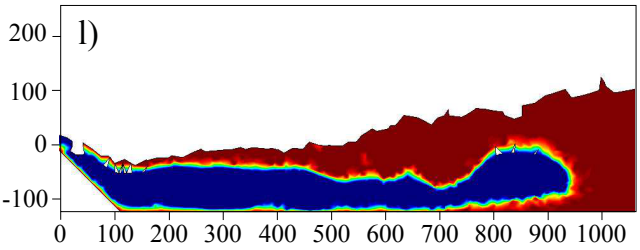

(b) PFEM

Figure 6. Lituya Bay landslide. Comparative snapshots of the experiment by Fritz et $a l$. [37] (left) and the results using PFEM and a variable-yield Bingham model (right). The first image was taken $11.25 \mathrm{~s}$ after impact. The time increment between images is $1.75 \mathrm{~s}$. 


\subsection{Lituya Bay landslide. Full-scale $3 D$ case study using the PFEM}

The PFEM was previously tested to model the Lituya Bay landslide using a full $3 D$ PFEM model [23]. The sliding mass was modelled as a quasi-incompressible viscous fluid. The maximum run-up obtained was $551 \mathrm{~m}$ in the Northern shore (5\% higher than the observed), and $195 \mathrm{~m}$ in the Southern shore (6 \% lower than the registered value). All the computational details and a comprehensive set of results using PFEM can be found in [23].

\subsection{Hypothetical landslide at Yesa Reservoir}

The example presented in this section refers to another full-scale test in a Spanish reservoir, in which the sliding mass was modelled as a Bingham fluid.

6.4.1. Description A slow landslide was observed in the southern margin of the Yesa reservoir in June 2006. This encouraged the dam owner to commission a study to delimit the characteristics of the wave that would be generated in the reservoir in case of occurrence. Hence, the model represents a hypothetical and highly unlikely event, given that the area is monitored and under strict control. The above-mentioned reference study [38] includes estimation on the volume and maximum velocity of the landslide in the worst possible scenario, taken into account the geotechnical and geological information available:

- The potentially unstable mass was estimated to be a 400-m-wide, 500-m-long block, with a maximum depth of $30 \mathrm{~m}$. The total estimated volume is $6 \mathrm{hm}^{3}$.

- The mean density was assumed to be $2,200 \mathrm{~kg} / \mathrm{m}^{3}$.

- The cross-section of the unstable mass, perpendicular to the landslide direction, was 12,000 $\mathrm{m}^{2}$.

- The maximum velocity of impact compatible with a reasonable value of the friction forces would be $3.3 \mathrm{~m} / \mathrm{s}$, and would be reached $25 \mathrm{~s}$ after the initiation of the event. These values were considered conservative, given that certain effects which would reduce the velocity at impact and the wave height were neglected. 
In summary, the landslide was considered as a rigid block falling into the reservoir with a fixed imposed velocity. This hypothesis is conservative and allowed to face the numerical model of the wave formation and propagation with simplified approaches. More precisely, in the previous study [38] the landslide was considered as a moving solid boundary and a commercial software which solved the shallow-water equations was used.

The application of simplified numerical models in this kind of analyses is frequently justified by the high computational cost of $3 D$ models. The aim of this work was to test whether the PFEM could provide a reasonable $3 D$ solution with moderate computational resources and computational time.

The mesh was created on the basis of the contour lines correspondent to the $1 / 5,000$ cartography. A Nurbs surface was generated using the pre/post-processing software GiD (www.gidhome.com). The geometry of the landslide was superimposed afterwards, together with the reservoir and the dam. In the Eastern boundary, the upstream reach of the reservoir was not taken into account. A vertical fixed boundary was located instead, which reflects the generated wave. Different mathematical tools can be used to avoid wave reflection, such as absorbing boundary conditions. These were not employed in this work, once confirmed that the effect of the reflected wave was negligible as regards to the main variable of interest, which is the maximum run-up at the dam.

A project for dam heightening is currently under development. The present numerical study considered the future geometry of the dam, with the foreseen dam crest height (520 m.a.s.1.) and normal operating level (511 m.a.s.1.). The computational domain was restricted to the part of the reservoir located between the landslide and the dam, together with the northern margin opposite to the potentially unstable area. The domain was similar to that considered in the reference study for the most detailed simulation, which was run with a shallow-water approach on a 10-m resolution model [38]. The $3 D$ PFEM model included a 2,300 x 2,700 $\mathrm{m}$ area in plan view (Figure 7), meshed with 4-noded tetrahedra with $10 \mathrm{~m}$ of average size. 
For the PFEM solution, the non-Newtonian Bingham fluid formulation described in this paper was chosen. The parameters were calibrated in $2 D$ simplified models, to ensure that the maximum velocity matched the estimate of the reference study $(3.3 \mathrm{~m} / \mathrm{s})$.
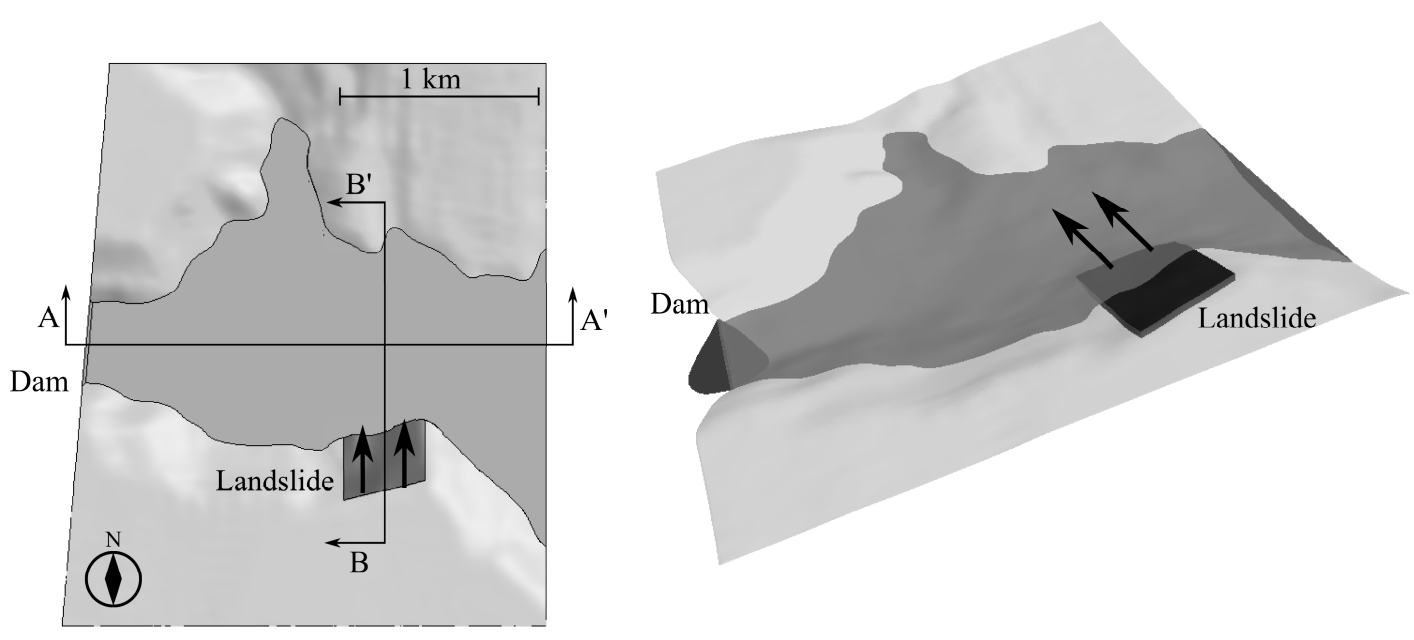

Figure 7. Landslide on Yesa reservoir. $3 D$ model geometry in plan view (left) and perspective (right)

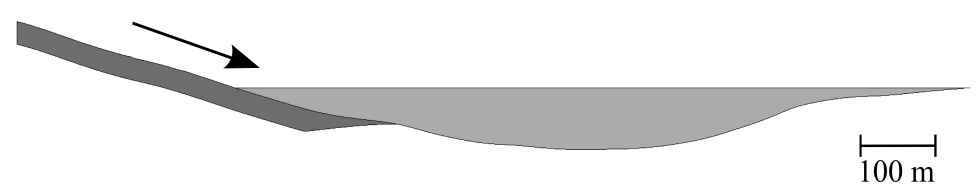

Figure 8. Landslide on Yesa reservoir. $2 D$ model used for calibrating the PFEM parameters. The figure corresponds to the B-B' section in Figure 7.

Previous numerical tests showed that the viscosity has little influence on the fluid kinematics, so it was set to a constant value (500 Pa.s). Both the yield stress and the regularisation parameter $m$ were varied (Table V) and the results were checked.

Figure 9 shows the PFEM results, in terms of the maximum velocity of the landslide. The influence of each parameter can be observed. A low value of the regularisation parameter results in a Newtoninan-fluid-like behaviour, with significant shear rate, even for low stress. On the contrary, large values of $m$ lead to a behaviour more similar to the bilinear law, and hence to a pure Bingham fluid. 
Table V. Values of yield stress and regularisation parameter for the calibration runs

\begin{tabular}{ccc}
\hline Case & Yield stress $(\mathrm{kPa})$ & $\mathrm{m}$ \\
\hline 1 & 50 & 5 \\
2 & 50 & 50 \\
3 & 50 & 500 \\
4 & 100 & 5 \\
5 & 100 & 50 \\
6 & 100 & 500 \\
7 & 150 & 5 \\
8 & 150 & 50 \\
9 & 150 & 500 \\
\hline
\end{tabular}
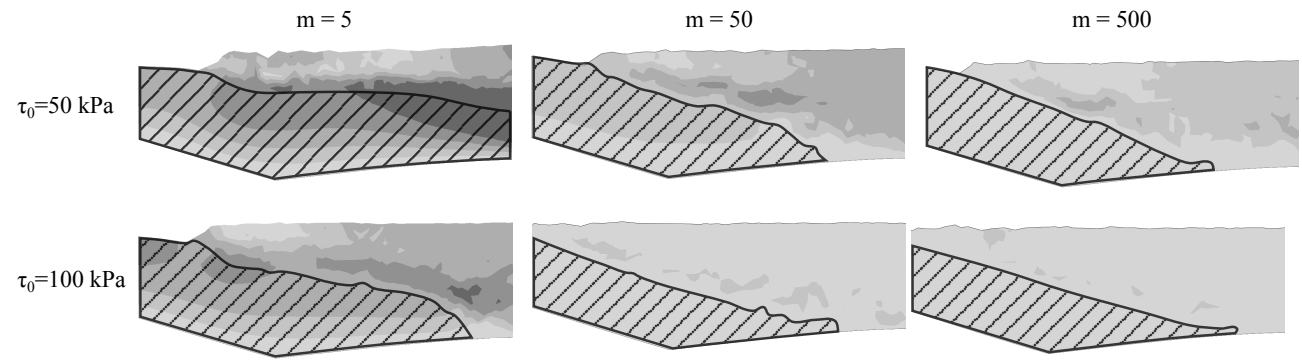

|Velocity
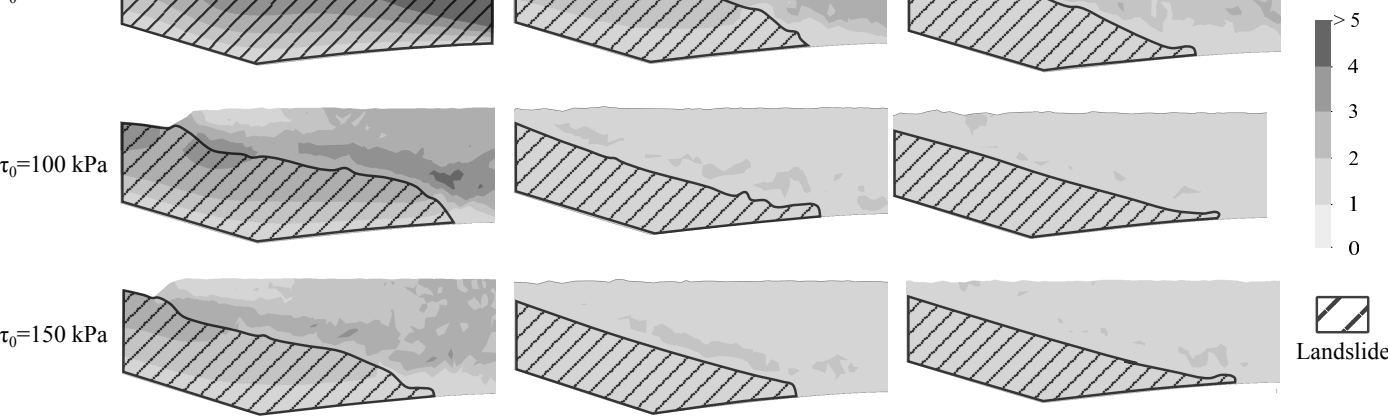

Figure 9. Landslide on Yesa reservoir. Velocity of landslide for different values of the parameters.

As regards to the yield stress, its value is inversely proportional to the mass stability: the greater the value, the lower the tendency to slide. A combination of a high yield stress and a high regularisation parameter results in a stable geometry, where no movement is registered (e.g. Case 9). 
It should be noted that the angle between the horizontal and the slip surface is moderate $\left(15^{\circ}\right)$, and that the toe of the slide lies below the initial reservoir level, hence the water mass contributes to its stability. Figure 9 shows that the maximum velocity of $3.3 \mathrm{~m} / \mathrm{s}$ is registered for Case 4 . Therefore, the $3 D$ model was run with $\tau_{0}=100 \mathrm{kPa}$ and $\mathrm{m}=5$.

6.4.2. PFEM results Some representative images of the results are included in Figure 10, where the plan view of the free surface location is plotted for different instants of the simulation. It can be observed that the wave propagates with a semi-circular front, centered at the impact point.

The free surface at various time steps was cut along an East-West axis ( $X$ in Figure 10 (a)) to take a closer look at the wave formation and propagation. The results are summarised in Figures 10 (b) and $10(\mathrm{c})$.
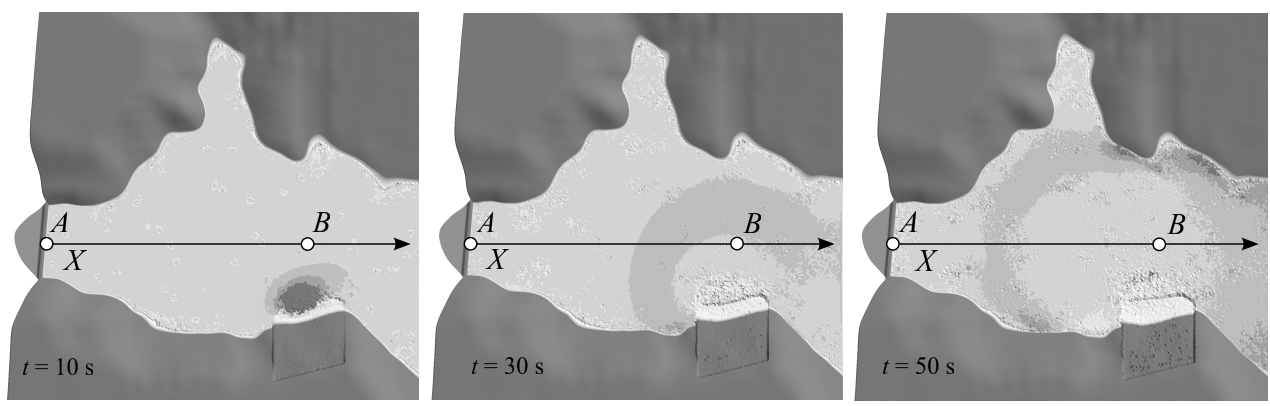

Free surface elevation (m.a.s.1.)

(a) Free surface evolution during wave generation and propagation. Plan view.

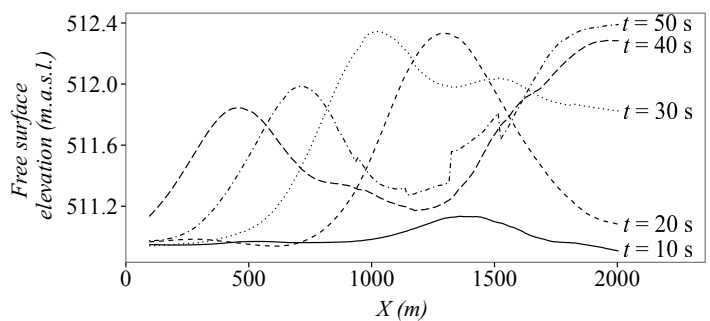

(b) Free surface profile along $X$ axis at different time steps

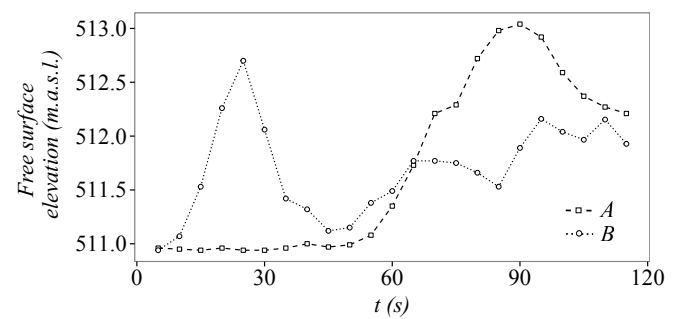

(c) Wave recorded at points $A$ and $B$

Figure 10. Landslide on Yesa reservoir. PFEM results.

The reference study [38] focused on the wave height at impact and the maximum run-up on the dam crest. Both values were extracted from the results of this work and are included in Table VI 
for comparison. Moreover, Figure 10 shows the time evolution of the free surface elevation at the referred locations.

Table VI. Hypothetical landslide at the Yesa reservoir. Comparison with the reference study.

\begin{tabular}{|c|c|c|c|c|}
\hline Study & Model & $\begin{array}{l}\text { Max. wave elevation } \\
\text { at impact (m) }\end{array}$ & $\begin{array}{l}\text { Max. run-up at the } \\
\qquad \operatorname{dam}(\mathrm{m})\end{array}$ & $\begin{array}{l}\text { Time of max. } \\
\text { run-up at the dam } \\
\text { (s) }\end{array}$ \\
\hline Ref. study [38] & $\begin{array}{l}\text { Commercial, } \\
\text { shallow-water }\end{array}$ & 1.93 & 2.24 & 105 \\
\hline This work & $3 D$, PFEM & 1.70 & 2.04 & 90 \\
\hline
\end{tabular}

Several conclusions can be drawn from the results:

- This case study can be analysed with a $2 D$ model, given that the slopes are moderate. In these situations, the error due to the $2 D$ simplification is low.

- A full 3D PFEM model can be applied to study real cases featuring several squared kilometres $\left(6.2 \mathrm{~km}^{2}\right.$ in this case), with moderate computational resources and time. Moreover, the PFEM provides a higher flexibility to model landslides of different kind, which cannot be considered as a fixed-velocity moving boundary.

- Results confirm the conclusions of the reference study: the potential consequences of the analysed hypothetical landslide are low.

\section{SUMMARY AND CONCLUSIONS}

Results of the application of the PFEM to model landslide-generated waves have been presented.

The cases analysed include laboratory tests with $2 D$ geometries as well as full-scale events, modelled in $3 D$. Likewise, slides of different nature were studied, both with respect to the rheology of the material (solid rigid, granular avalanche or landslide) and the sliding velocity (from 3 to 100 
$\mathrm{m} / \mathrm{s}$ ). The flexibility of the PFEM was exploited to reproduce the characteristics of each case, by means of utilising the most appropriate constitutive law.

It should be considered that the work deals with a highly complex phenomenon, with a great uncertainty about several of the variables involved, such as the geometry and friction of the sliding surface and the mechanics of the mobilised material.

The physical and numerical models used to analyse these events shall rely on simplifications of the real case, so results must be analysed cautiously, as estimates of the possible consequences in case of occurrence of the event. As an example, in the cases presented in this work the effect of the air was not taken into account, and the irregularities of the terrain were simplified.

In spite of these considerations, the results presented show that the PFEM is a useful tool for analysing landslide events, and can be used to estimate the consequences of landslide-generated waves at full scale in $3 D$. In addition, the variety of constitutive laws implemented allows us to adapt the model to the geological-geotechnical features of each particular case, ranging from the more fluid-like landslides to the fall of rigid solids.

In particular, the variable-yield Bingham model used in this work reduces the uncertainty on the definition of the numerical parameters of the sliding mass and avoids the need for running calibration tests. The model is defined on the basis of the internal friction angle of the material, which is a commonly used property and easy to estimate on-site.

The authors are currently working on the optimisation of the method to face problems on larger domains or with finer meshes. Likewise, the erosion module [19] is being tested to study phenomena such as the mass that superficial erosion incorporates to the slide during the fall, or the damages to earth dams in case of overtopping [39] and the effect of the air in the landslide motion, among others.

\section{ACKNOWLEDGEMENTS}

The authors wish to thank Mr. Raimundo Lafuente and Mr. René Gómez, from the Ebro River Basin Authority (Confederación Hidrográfica del Ebro, CHE) for providing the information for modelling the 
Yesa case study. The research was supported by the Spanish Ministry of Economy and Competitiveness (Ministerio de Economía y Competitividad, MINECO) through the project Xlide (IPT-2011-1287-370000). This work was also supported by the ERC Advanced Grant project SAFECON (AdG-267521) of the European Research Council.

\section{REFERENCES}

[1] The Particle Finite Element Method - PFEM. URL http://www.cimne.com/pfem/.

[2] Pastor M, Blanc T, Haddad B, Drempetic V, Morles MS, Dutto P, Stickle MM, Mira P, Merodo JF. Depth averaged models for fast landslide propagation: mathematical, rheological and numerical aspects. Archives of Computational Methods in Engineering 2014; :1-38.

[3] Vacondio R, Mignosa P, Pagani S. 3D SPH numerical simulation of the wave generated by the Vajont rockslide. Advances in Water Resources 2013; 59:146-156.

[4] Alonso EE, Pinyol NM. Landslides in reservoirs and dam operation. Dam Maintenance and Rehabilitation II. CRC Press, 2011; 3-27.

[5] Clague J, Stead D. Landslides: Types, Mechanisms and Modeling. Cambridge University Press, 2012.

[6] Pastor M, Blanc T, Pastor MJ. A depth-integrated viscoplastic model for dilatant saturated cohesivefrictional fluidized mixtures: application to fast catastrophic landslides. Journal of Non-Newtonian Fluid Mechanics 2009; 158(1-3):142-153.

[7] Pastor M, Haddad B, Sorbino G, Cuomo S, Drempetic V. A depth-integrated, coupled SPH model for flow-like landslides and related phenomena. International Journal for Numerical and Analytical Methods in Geomechanics 2009; 33(2):143-172.

[8] Bosa S, Petti M. Shallow water numerical model of the wave generated by the Vajont landslide. Environmental Modelling \& Software 2011; 26(4):406-418.

[9] Ward SN, Day S. Tsunami balls: a granular approach to tsunami runup and inundation. Communications in Computational Physics 2008; 3:222-249.

[10] Ward SN, Day S. The 1958 Lituya bay landslide and tsunami - a tsunami ball approach. Journal of Earthquake and Tsunami Dec 2010; 4(4):285-319.

Int. J. Numer. Anal. Meth. Geomech. (2010)

Prepared using nagauth.cls

DOI: $10.1002 / \mathrm{nag}$ 
[11] Oñate E, Celigueta MA, Idelsohn SR, Salazar F, Suárez B. Possibilities of the particle finite element method for fluid-soil-structure interaction problems. Computational Mechanics 2011; 48(3):307-318.

[12] Larese A. A coupled Eulerian-PFEM model for the simulation of overtopping in rockfill dams. Phd thesis: Universitat Politècnica de Catalunya (UPC BarcelonaTech), Barcelona, Spain, 2012. URL http://hdl.handle. net/10803/108502.

[13] Oñate E, Celigueta MA, Latorre S, Casas G, Rossi R, Rojek J. Lagrangian analysis of multiscale particulate flows with the particle finite element method. Computational Particle Mechanics 2014; 1(1):85-102.

[14] Oñate E, Idelsohn S. The particle finite element method. an overview. International Journal of Computational Methods 2004; 1:267-307.

[15] Larese A, Rossi R, Oñate E, Idelsohn SR. Validation of the particle finite element method (PFEM) for simulation of free surface flows. Engineering Computations May 2008; 25(4):385-425.

[16] Idelsohn S, Oñate E, Del Pin F. A lagrangian meshless finite element method applied to fluid-structure interaction problems. Computers and Structures May 2003; 81(8):655-671.

[17] Idelsohn S, Oñate E, Pin FD. The particle finite element method: a powerful tool to solve incompressible flows with free-surfaces and breaking waves. International Journal for Numerical Methods in Engineering 2004; 61(7):964-989.

[18] Aubry R, Idelsohn S, Oñate E. Particle finite element method in fluid-mechanics including thermal convection-diffusion. Computers \& Structures Jun 2005; 83(17-18):1459-1475.

[19] Oñate E, Idelsohn SR, Celigueta MA, Rossi R. Advances in the particle finite element method for the analysis of fluid-multibody interaction and bed erosion in free surface flows. Computer Methods in Applied Mechanics and Engineering Mar 2008; 197(19-20):1777-1800.

[20] Larese A, Rossi R, Oñate E. Coupling eulerian and lagrangian models to simulate seepage and evolution of failure in prototype rockfill dams. Proceeding of the XI ICOLD Benchmark Workshop on Numerical Analysis of Dams, SpanCOLD, Madrid, Spain 2011; ISBN: 978-84-695-1816-8.

[21] Larese A, Rossi R, Oñate E, Idelsohn S. A coupled PFEM-eulerian approach for the solution of porous FSI problems. Computational Mechanics 2012; 50(6):805-819. 
[22] Zhang X, Krabbenhoft K, Sheng D, Li W. Numerical simulation of a flow-like landslide using the particle finite element method. Computational Mechanics 2014; :1-11doi:10.1007/ s00466-014-1088-z. URL http://dx.doi.org/10.1007/s00466-014-1088-z.

[23] Salazar F, Oñate E, Morán R. Numerical modelling of landslides in reservoirs via the particle finite element method (pfem). Revista Internacional de Métodos Numéricos para Cálculo y Diseño en Ingeniería 2012; 28(2):112-123. [in Spanish].

[24] Cremonesi M, Frangi A, Perego U. A lagrangian finite element approach for the simulation of waterwaves induced by landslides. Computers \& Structures 2011; 89(11):1086-1093.

[25] Quecedo M, Pastor M, Herreros M, Fernández Merodo J. Numerical modelling of the propagation of fast landslides using the finite element method. International Journal for Numerical Methods in Engineering 2004; 59(6):755-794.

[26] Oñate E, Zarate F, Miquel J, Santasusana M, Celigueta M, Arrufat F, Gandikota R, Valiullin K, Ring L. A local constitutive model for the discrete element method. application to geomaterials and concrete. Submitted to: Computational Particle Mechanics 2015; .

[27] Zienkiewicz O, Jain P, Onate E. Flow of solids during forming and extrusion: Some aspects of numerical solutions. International Journal of Solids and Structures 1978; 14(1):15-38.

[28] Chhabra RP. Non-newtonian fluids: An introduction. Rheology of Complex Fluids, Krishnan JM, Deshpande AP, Kumar PBS (eds.). Springer New York, 2010; 3-34.

[29] Papanastasiou TC. Flows of materials with yield. Journal of Rheology 1987; 31(5):385-404.

[30] Zienkiewicz O, Taylor R, Zhu J. The finite element method: its basis and fundamentals. ButterworthHeinemann, 2005.

[31] Codina R. Stabilization of incompressibility and convection through orthogonal sub-scales in finite element method. Computer Methods in Applied Mechanics and Engineering 2000; 190:1579-1599.

[32] Wood W, Bossak M, Zienkiewicz O. An alpha modification of Newmark's method. Int. J. for Num. Meth in Eng. 1980; 15:1562-1566.

[33] Adam D, Wood W. Comparison of Hilber-Hughes-Taylor and Bossak $\alpha$ methods for the numerical integration of vibration equations. International Journal for Numerical Methods in Engineering 1983; 19:765-771. 
[34] Calvo N. Generación de mallas tridimensionales por métodos duales. $\mathrm{PhD}$ thesis: Univesidad Nacional del Litoral, Argentina, 2005. [in Spanish].

[35] Sælevik G, Jensen A, Pedersen G. Experimental investigation of impact generated tsunami; related to a potential rock slide, Western Norway. Coastal Engineering Sep 2009; 56(9):897-906.

[36] Quecedo M, Pastor M, Herreros MI. Numerical modelling of impulse wave generated by fast landslides. International Journal for Numerical Methods in Engineering 2004; 59(12):1633-1656.

[37] Fritz HM, Mohammed F, Yoo J. Lituya bay landslide impact generated mega-tsunami 50th anniversary. Pure and Applied Geophysics 2009; 166(1-2):153-175.

[38] Garrote L, Laguna F. Studies on the ondulatory movements generated in reservoirs in the hypothesis of landslides. Technical Report, UPM 2008. [in Spanish].

[39] Larese A, Rossi R, Oñate E, Toledo M, Moran R, Campos H. Numerical and experimental study of overtopping and failure of rockfill dams. International Journal of Geomechanics (ASCE) 2013; doi: 10.1061/(ASCE)GM.1943-5622.0000345. 